sites and relation to clinical characteristics, laboratory features and disease activity.

Patients and methods Medical records of 250 Egyptian SLE patients attending the Rheumatology department, Cairo University hospitals were reviewed retrospectively for the clinical and laboratory features, SLE disease activity index (SLEDAI) and treatment received.

Results Infection was found in 119 (47.6\%) patients, with bacterial infection being the commonest in 99 (83\%) followed by fungal infection in $30(25 \%)$ and viral infection in 22 (18.5\%). The commonest site of infection was the skin (37\%) followed by the urinary tract (31\%) and chest (19\%). In SLE patients with infection there was a significant increase in the frequency of malar rash $(p=0.001)$, photosensitivity $(p=0.01)$, oral ulcers $(p<0.001)$, alopecia $(p=0.017)$ and Raynauds $(p=0.017)$ compared to those without infection. Pulmonary and neuropsychiatric manifestations were also significantly increased in those with infection $(p=0.001$ and $p<0.001)$. A significantly higher number of patients with infection were receiving pulse steroids $(\mathrm{p}=0.016)$, cyclophosphamide $(p=0.011)$ and a higher oral prednisolone dose $(p=0.03)$. The SLEDAI was significantly higher $(26.02 \pm 8.23)$ in those with infection compared to those without $(15.57 \pm 6.43)(\mathrm{p}<0.001)$. $\mathrm{C}$-reactive protein (CRP) was significantly higher in those with infection $(p<0.001)$. On performing a logistic regression analysis, only SLEDAI $(\mathrm{p}<0.001)$ and CRP $(\mathrm{p}<0.001)$ were significant predictors of infection.

Conclusion Disease activity and CRP are important predictors for infection in SLE patients.

\section{PS3:56 THE ASSOCIATION BETWEEN SELF-REPORTED PHYSICAL ACTIVITY ON DISEASE STATUS PATIENTS WITH SYSTEMIC LUPUS ERYTHEMATOSUS: DATA FROM KOREAN LUPUS NETWORK (KORNET) REGISTRY}

S Kim, J Choe, S Park, H Lee. Catholic University of Daegu School of Medicine, Daegu, South Korea

\subsection{6/lupus-2018-abstract.103}

Objective The aim of this study was to identify the influence of physical activity on disease activity and damage in systemic lupus erythematosus (SLE).

Methods A total of 464 patients with Sjögren's syndrome were consecutively enrolled from KORean lupus Network (KORNET) registry. This registry assessed clinical features, disease activity (Systemic Lupus Erythematosus Disease Activity Index 2000 [SLEDAI-2K]), and disease damage (Systemic Lupus International Collaborating Clinics/American College of Rheumatology [SLICC/ACR] damage index) at the enrollment of study. Self-reported physical activity was measured by international physical activity questionnaire (IPAQ). Statistical analyses were used by Spearman's correlation and Mann-Whitney U test.

Results The median total physical activity (MET-minute/day) was 1173.0 (IQR 396.0-2772.0). There is significant difference of vigour activity between patients with lupus nephritis $(n=110)$ and without lupus nephritis $(n=354)(p=0.048)$, but not total, moderate, and walking activities. Among total patients, total IPAQ score was marginally associated with SLEDAI and SLICC/ACR scores $(r=-0.142, \quad \mathrm{p}=0.008$ and $r=-0.104, p=0.026)$. Higher SLICC/ACR scores was associated with lower walking activity and total activity of IPAQ in patients with lupus nephritis, $(\mathrm{r}=-0.256, \mathrm{p}=0.007$ and $\mathrm{r}=-0.193, \mathrm{p}=0.044$, respectively).

Conclusion This study showed that self-reported physical activity might be in part associated with disease activity and damage in patients with SLE.

\section{PS3:57 IMPACT OF LUPUSNEPHRITIS ON MORTALITY IN SYSTEMIC LUPUS ERYTHEMATOSUS. A POPULATION BASED COHORT FROM NORWAY}

${ }^{1}$ SER Moe, ${ }^{1} \varnothing$ Molberg, ${ }^{2} \mathrm{EH}$ Strøm, ${ }^{1} \mathrm{~K}$ Lerang. 'Department of Rheumatology, Os/o University Hospital, Rikshospitalet, Oslo, Norway: ${ }^{2}$ Department of Pathology, Oslo University Hospital, Rikshospitalet, Oslo, Norway

\subsection{6/lupus-2018-abstract.104}

Objective Recent data has shown that patients with lupus nephritis (LN) have increased mortality. However, no studies have been population based, and few compare mortality data in Systemic Lupus Erythematosus (SLE) patients with and without LN. The aim of our study was to investigate mortality in patients who does or does not develop LN in a population based cohort.

Methods Multiple sources were used to identify all SLE patients in Oslo during 1999-2009 who met 4 or more of the American College of Rheumatology (ACR) criteria. Follow up time was until 1 st January 2014. Presence of LN was defined by the ACR criteria. Standardised mortality ratio (SMR) was compared to observed deaths in a matched control population.

Results Of the 325 SLE patients included in this study, 98 (30\%) developed LN. 75 patients $(77 \%)$ had biopsy proven LN. A total of 56 deaths occurred during the study period, corresponding to an overall SMR for all SLE patients of 2,1 (95\% confidence intervall (CI) 1,2 to 3,4). The SMR estimate for LN patients were 3,8 (95\% CI: 2,1 to 6,2$)$ and $1,7(95 \%$ CI: 0,9 to 2,7$)$ for non $\mathrm{LN}$ patients.

Conclusion LN is associated with increased mortality, however SLE patients who do not develop LN have a good overall prognosis with no significant higher mortality than the general population.

\section{PS3:58 LIPID PROFILE CHARACTERISATION IN PATIENTS WITH JUVENILE SLE WITH AND WITHOUT LUPUS NEPHRITIS - EXPERIENCE OF A PORTUGUESE CENTRE}

\begin{abstract}
${ }^{1,2} \mathrm{~A}$ Águeda, ${ }^{3} \mathrm{M}$ Guerra, ${ }^{4} \mid$ Jorge, ${ }^{2,5} \mathrm{R}$ Ferreira, ${ }^{2,6} \mathrm{M}$ Rodrigues, ${ }^{2,6}$ । Brito. ${ }^{1}$ Rheumatology, Centro Hospitalar do Baixo Vouga E.P.E., Aveiro, Portugal; ${ }^{2}$ Faculty of Medicine of Porto University, Porto, Portugal; ${ }^{3}$ Rheumatology, Centro Hospitalar Vila Nova de Gaia/Espinho, Porto, Portugal; ${ }^{4}$ Physical Medicine and Rehabilitation, Centro Hospitalar do Porto, Portugal; ${ }^{5}$ Rheumatology, Centro Hospitalar de São João, Porto, Portugal; ${ }^{6}$ Pediatric Rheumatology Unit, Porto, Portugal

\subsection{6/lupus-2018-abstract. 105}

Introduction SLE is a multisystem chronic inflammatory disease and has been associated with premature atherosclerosis and so, controlling classic cardiovascular (CV) risk factors is crucial. Dyslipidaemia is an important $\mathrm{CV}$ risk factor and has been found to be altered in SLE patients.

Purpose Compare lipid profiles of two groups of Juvenile SLE patients, without Lupus Nephritis (group 1) and with Lupus Nephritis (group 2). Verify factors that might correlate with abnormalities in lipid profile. 
Abstract PS3:57 Table 1 Patient characteristic in SLE patients with and without lupus nephritis (LN)

\begin{tabular}{|c|c|c|c|c|}
\hline & All & $\underline{L N}$ & Non LN & p value \\
\hline & $\mathrm{N}=325$ & $\mathrm{~N}=98(30)$ & $N=227(70)$ & \\
\hline Female & $291(90)$ & $86(86)$ & $205(90)$ & ns \\
\hline Of non European descent & $54(17)$ & $26(48)$ & $28(12)$ & 0,013 \\
\hline Age at diagnosis & $32(7-83)$ & $22(7-82)$ & $37(10-83)$ & $<0,001$ \\
\hline Age at first symptom & $28(7-82)$ & $21(7-82)$ & $32(9-71)$ & $<0,001$ \\
\hline Age at study end * & $52(20-99)$ & $42(20-89)$ & $58(22-99)$ & $<0,001$ \\
\hline Disease duration** & $14(1-49)$ & $19(1-47)$ & $15(2-49)$ & ns \\
\hline \multicolumn{5}{|l|}{ Antibody profile } \\
\hline Anti-DNA ab & $205(63)$ & $80(84)$ & $125(55)$ & $<0,001$ \\
\hline Anti - SM ab & $48(15)$ & $24(24)$ & $24(11)$ & 0,027 \\
\hline Anti-Cardiolipin ab & $96(30)$ & $24(24)$ & $72(32)$ & ns \\
\hline LA & $88(27)$ & $32(33)$ & $56(25)$ & ns \\
\hline Anti-SSB ab & $61(19)$ & $10(10)$ & $51(22)$ & 0,006 \\
\hline Anti - SSA ab & $136(42)$ & $32(33)$ & $104(46)$ & ns $(0,051)$ \\
\hline
\end{tabular}

Abstract PS3:57 Table 2 Standardised mortality rate (SMR), total and stratified by age at death

\begin{tabular}{ccccccc}
\multicolumn{1}{c}{ LN } & \multicolumn{5}{c}{ Non LN } \\
\hline & $\begin{array}{c}\mathrm{N}, \\
\text { deaths }\end{array}$ & SMR & CI & $\begin{array}{c}\text { N, } \\
\text { deaths }\end{array}$ & SMR & CI \\
\hline Age group: & & & & & & \\
$16-39$ & 6 & 13,4 & $7,2-22,1$ & 1 & 1,6 & $0,2-5,3$ \\
$40-59$ & 2 & 2,2 & $0,5-5,5$ & 11 & 2,2 & $1,1-3,9$ \\
$60-79$ & 6 & 4,9 & $3,8-6,1$ & 18 & 1,8 & $1,0-2,8$ \\
$80 \geq$ & 2 & 5,2 & $4,5-6,0$ & 10 & 1,6 & $1,2-2,0$ \\
Total & 16 & $\mathbf{3 , 8}$ & $\mathbf{2 , 1 - 6 , 2}$ & 40 & $\mathbf{1 , 7}$ & $\mathbf{0 , 9 - 2 , 7}$ \\
\hline
\end{tabular}

$\mathrm{N}$ : Number, $\mathrm{Cl}: 95 \%$ confidence interval

LN = lupus nephritis, defined after ACR criteria

Methods Retrospective analysis of the lipid profile (Total cholesterol, LDL, HDL and triglycerides) of two groups of patients with juvenile SLE, with and without lupus nephritis (LN), in two points in time, when $\mathrm{LN}$ was first diagnosed and 6-12 months after initiating LN treatment.

Statistical analysis was performed using Student t-test and Pearson correlation coefficient test. Significance level was set as $<0.05$. Results We included 27 patients with Juvenile SLE, 17 with LN (82,4\% Class IV; $17.6 \%$ class III). There were no differences between groups in baseline characteristics. Mean present age was $27.1 \pm 6.4$ years for group $1(n=10)$ and $27.6 \pm 6.1$ years for group $2(n=17)$.

Analysis of lipid profile showed higher mean values of TC, LDL and Triglycerides in group 2 at both time points (table 1), however the differences between groups did not reach statistical significance.

On a sub-analysis of group 2, we studied the correlation between lipid profile and proteinuria. No correlation was found at time 1 however, at time 2 a strong positive correlation was found between proteinuria values and TC and 
Abstract PS3:58 Table 1 Mean values of lipid profile at time 1 and 2 (result units in $\mathrm{mg} / \mathrm{dl}$ )

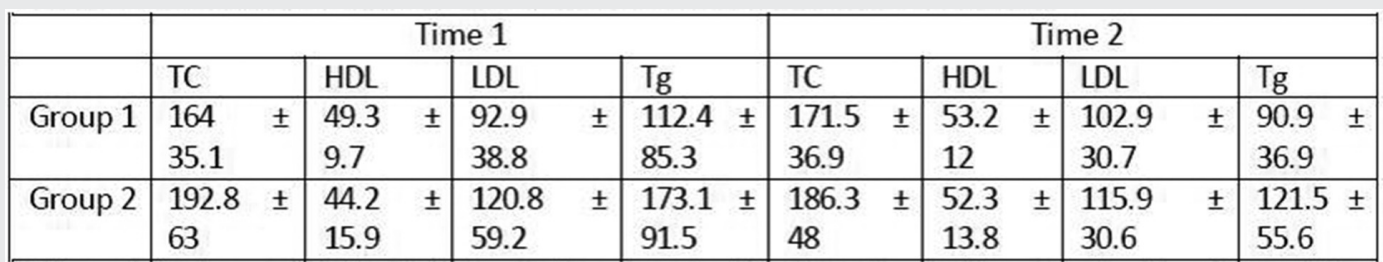

TC: total cholesterol; HDL: high densitylipoprotein; LDL: low-density lipoprotein; Tg: triglycerides

between proteinuria and LDL levels $(r=0.67, p=0.017$ and $\mathrm{r}=0.74, \mathrm{p}=0.009$ respectively).

Conclusion Our study shows that Juvenile SLE patients with LN tend to have more abnormalities of lipid profile than patients without LN, namely with higher TC, LDL and Tg, and lower HDL.

A significant positive correlation was found at time 2 between proteinuria and TC and LDL levels, reflecting that the severity of proteinuria correlates with abnormalities in lipid profile.

These results reinforce that juvenile SLE population, namely with $\mathrm{LN}$, should have their CV risk factors, such as lipid profile, carefully monitored.

\section{PS3:59 IS THERE A DIFFERENCE BETWEEN LIPID PROFILE BEFORE AND AFTER LUPUS NEPHRITIS TREATMENT IN PATIENTS WITH JUVENILE SYSTEMIC LUPUS ERYTHEMATOSUS?}

II Santos Jorge, ${ }^{2} \mathrm{M}$ Guerra, ${ }^{3} \mathrm{~A}$ Águeda, ${ }^{4,5} \mathrm{R}$ Ferreira, 5,6 M Rodrigues, ${ }^{5,6}$ | Brito. ${ }^{1}$ Physical Medicine and Rehabilitation Department, Centro Hospitalar do Porto, Portugal; ${ }^{2}$ Rheumatology Department, Centro Hospitalar Vila Nova de Gaia/Espinho, Vila Nova de Gaia, Portugal; ${ }^{3}$ Rheumatology Department, Centro Hospitalar do Baixo Vouga, Aveiro, Portugal; ${ }^{4}$ Rheumatology Department, Centro Hospitalar de São João, Porto, Portugal; ${ }^{5}$ Faculty of Medicine of Porto University, Porto, Portugal; ${ }^{6}$ Paediatric Rheumatology Unit, Centro Hospitalar de São João, Porto, Portugal

\subsection{6/lupus-2018-abstract.106}

Purpose Renal involvement is one of the major determinants of outcome in patients with Systemic Lupus Erythematosus (SLE). Dyslipidaemia occurs frequently in Juvenile SLE. This study aimed to determine if there were differences in lipid profile in Juvenile SLE patients with lupus nephritis (LN) in two time points, time 1 at $\mathrm{LN}$ diagnosis and time 2 after 612 months of treatment.

Methods Retrospective analysis of the lipid profile of a population of Juvenile SLE patients with $\mathrm{LN}$ in two time points. Lipid profile evaluation included total cholesterol (TC), triglycerides (TG), high density lipoprotein (HDL) and low-density lipoprotein (LDL). Statistical analysis was performed using $\mathrm{T}$ Student Test. Significance level was set as $<0.05$.

Results At LN diagnosis, time 1, the mean TC was $198,1 \mathrm{mg} /$ dL ( $\pm 62,9)$, TG was $175,1 \mathrm{mg} / \mathrm{dL}( \pm 89,5)$, HDL was $44,3 \mathrm{mg} /$ $\mathrm{dL}( \pm 16,9)$ and $\mathrm{LDL}$ was $120,6 \mathrm{mg}( \pm 61,2)$. At time 2 , the mean TC was $188,2 \mathrm{mg} / \mathrm{dL}( \pm 47.0)$, TG was $126,3 \mathrm{mg} / \mathrm{dL}$ $( \pm 57.1)$, HDL was $52,3 \mathrm{mg} / \mathrm{dL}( \pm 13,8)$ and LDL was $115,9 \mathrm{mg} / \mathrm{dL}( \pm 30,6)$.$) . Despite higher levels of TC, TG and$ LDL levels at time one, the variation of lipid profile between the two time points did not reach statistical significance.
Conclusions Our study showed that the lipid profile of LN patients tends to improve with LN treatment, as showed by the lower levels of TC, TG and LDL and higher levels of HDL observed at time 2 .

\section{PS3:60 INCIDENCE OF VASCULITIS IN HOSPITALISED LUPUS PATIENTS}

${ }^{1} \mathrm{Z}$ Mirfeizi, ${ }^{2} \mathrm{ZH}$ Shariati Sarabi, ${ }^{3} \mathrm{E}$ Atabati, ${ }^{4} \mathrm{R}$ Ranjbar, ${ }^{1} \mathrm{M}$ Sahebari, ${ }^{5} \mathrm{~K}$ Hashemzadeh. ${ }^{1}$ Associate Professor of Rheumatology, Faculty of Medicine, Mashhad University of Medical Sciences, Mashhad, Iran; ${ }^{2}$ Professor of Rheumatology, Faculty of Medicine, Mashhad University of Medical Sciences, Mashhad, Iran; ${ }^{3}$ Assistant Professor of Rheumatology, Faculty of Medicine, Birjand University of Medical sciences, Birjand, Iran; ${ }^{4}$ Emergency Physician Specialist, Faculty of Medicine, Mashhad University of Medical Sciences, Mashhad, Iran; ${ }^{5}$ Assistant Professor of Rheumatology, Faculty of Medicine, Mashhad University of Medical Sciences, Mashhad, Iran

\subsection{6/lupus-2018-abstract. 107}

Introduction Systematic Lupus Erythematosus (SLE) is an autoimmune disease which is associated with multiple target organ damage. Multiple hospitalizations can occur during their lifelong. Systemic vasculitis manifestation is one of the most important reasons leading to refer to rheumatology clinics. The aim of this study is to evaluate different picture of systemic vasculitis in lupus patients.

Materials and methods Eighty-one known SLE patients based on 2012 Revised Criteria who were visited in Rheumatology Department of Imam Reza Hospital from January 2012 to December 2014 having undergone a thorough physical examination by rheumatologist, classified based on demographic and SLE-related vasculitis manifestations. We focused on vasculitis manifestation in this group of patient.

Results In total $17.3 \%$ of our lupus patients were diagnosed as vasculitis. In the vasculitis group cutaneous vasculitis (50\%), thromoembolic events $(7 / 1 \%)$, cerebral vasculitis $(7 / 1 \%)$, retinal vasculitis $(7 / 1 \%)$ and aortic involvement were detected. Systemic vasculitis is the second most common causes for referring to the hospital. Mean SLEDAI and SLICC scores were $22.79( \pm 13.29)$ and $1.95( \pm 1.46)$, in vasculitis group respectively.

Considering SLICC SLE Criteria, prevalence of vasculitis lesion were found more often in ocular, renal, gastrointestinal and CNS system $(\mathrm{p}=0.03,0.01,<0.001,<0.03$ respectively.

Conclusion Vasculitis manifestation in lupus probably is one of the most important and life threatening complication which may have brought these patients to rheumatology centre. In this study, we found that vasculitis may be the first presentation of SLE patients with severe organ damage with no previous history of this disease. 